Received: 28 January 2019

Accepted: 17 April 2019

Published online: 01 May 2019

CIENTIFIC REP

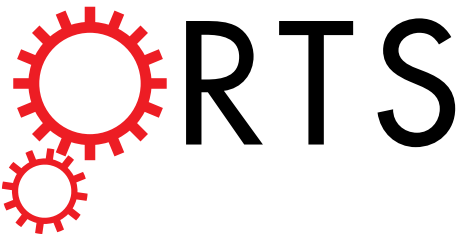

Corrected: Author Correction

\title{
OPEN Transcription Factor HOXA9 is Linked to the Calcification and Invasion of Papillary Thyroid Carcinoma
}

Yilan Jin ${ }^{1}$, Hyeung Kyoo Kim², Jeonghun Lee ${ }^{2}$, Euy Young Soh ${ }^{2}$, Jang-Hee Kim³ ${ }^{3}$ Insun Song ${ }^{1,4}$, Yoon-Sok Chung ${ }^{1}$ \& Yong Jun Choi $\mathbb{C}^{1}$

Calcification is important for the diagnosis of papillary thyroid carcinoma (PTC). Runt-related transcription factor 2 (RUNX2), a master transcription factor associated with osteogenic differentiation, is reportedly related to PTC calcification and invasiveness. However, its regulatory role in this process is somewhat uncharacterized. Here, we attempted to identify genes that regulate RUNX2 and clarify its function in PTC carcinogenesis and calcification. The expression of RUNX2-upstream genes was evaluated by real-time PCR in Nthy-Ori 3-1 normal thyroid cells and TPC1 and BHP10-3 PTC cell lines. Luciferase and chromatin immunoprecipitation assays were performed with candidate genes after cloning the RUNX2 promoter. We found that RUNX2 promoter activity was enhanced by homeobox family A9 (HOXA9). Over-expression of HOXA9 was found to enhance alkaline phosphatase activity, mineralization, and in vitro tumour cell migration and invasion, whereas downregulation had the opposite effects. These results indicate that HOXA9, a positive regulator of RUNX2, can enhance calcification, migration, and invasion in PTC. Our data improve the understanding of the molecular mechanisms of microcalcification in PTC as well as tumorigenesis.

Papillary thyroid carcinoma (PTC) is the most common type of malignant thyroid tumour ${ }^{1-3}$. Calcifications, characteristic of this disease, are important for the diagnosis of $\mathrm{PTC}^{4,5}$. However, these are frequently found not only in cancer tissues but also in some benign nodules ${ }^{6}$. Psammoma bodies (PBs), concentric lamellated calcified structures, are most common in histological sections of $\mathrm{PTC}^{7,8}$. PBs are largely considered the result of dystrophic calcification, in which deposition occurs locally in nonviable or dying tissues ${ }^{9}$. However, rather than being the outcome of dystrophic calcification of dead or dying tissue, it has been suggested that PBs might represent an active biologic process leading to the degeneration/death of tumour cells and the retardation of growth of the neoplasm ${ }^{9}$. Thus, the underlying mechanism and role of calcification in PTC are not fully understood.

Runt-related transcription factor 2 (RUNX2) belongs to the RUNX family and consists of three isoforms, namely types I, II, and III ${ }^{10}$. The main function of type I and II RUNX2 is as transcription factors involved in osteoblast differentiation and bone formation ${ }^{11,12}$. However, they are also involved in the carcinogenesis of breast and prostate cancers, as well as bone metastasis ${ }^{13,14}$. Moreover, some findings have suggested that high RUNX2 expression can predict breast cancer recurrence ${ }^{15}$ or induce myeloma progression in the bone ${ }^{16}$. RUNX2 also plays a critical role in mesenchymal cell differentiation to the osteogenic lineage ${ }^{17}$.

Recently, it has been reported that thyrocytes show similar characteristics to osteoblasts and that the expression of RUNX2 is increased in PTC tissues ${ }^{18}$. Further, PTC patients with microcalcifications were found to express significantly higher levels of RUNX2 mRNA in serum than those without microcalcifications ${ }^{17}$. Enhanced RUNX2 signalling has been functionally linked to tumour invasion and metastasis in thyroid carcinoma by regulating epithelial-to-mesenchymal transition-related molecules, matrix metalloproteinases, and angiogenic/

\footnotetext{
${ }^{1}$ Department of Endocrinology and Metabolism, Ajou University School of Medicine, Suwon, South Korea. ${ }^{2}$ Department of Surgery, Ajou University School of Medicine, Suwon, South Korea. ${ }^{3}$ Department of Pathology, Ajou University School of Medicine, Suwon, South Korea. ${ }^{4}$ School of Biological Sciences, Seoul National University, Seoul, South Korea. Correspondence and requests for materials should be addressed to Y.J.C. (email: colsmile@hanmail. net)
} 
lymphangiogenic factors ${ }^{19}$. However, the regulatory role of RUNX2 in thyroid calcification and carcinogenesis has not been fully elucidated.

In this study, our aim was to discover a novel protein that regulates the expression of RUNX2 and to clarify the function of this marker and RUNX2 in carcinogenesis and calcification. For this, we screened several candidate transcription factors, upstream genes of RUNX2, and homeobox A9 (HOXA9) was identified as a potential candidate. Hox proteins, a group of homeodomain-containing transcription factors, play a key role in oncogenesis and are extremely dysregulated both in solid and haematological malignancies ${ }^{20-22}$. The expression of HOXA9, as a member of the HOX gene family, is usually altered in solid cancers ${ }^{23}$. Thus, we elucidated the relationship between HOXA9 and RUNX2 and the associated functions in PTC carcinogenesis and calcification.

\section{Results}

HOXA9 regulates RUNX2 gene expression. To discover a novel protein that regulates the expression of RUNX2, we selected seven candidates (catenin beta interacting protein 1 (CTNNBIP1), distal-less homeobox 3 (DLX3), HOXA9, NK2 homeobox 5 (NKX2-5), $\rightarrow$ NK3 homeobox 2 (NKX3-2), runt related transcription factor 1 (RUNX1), and SRY-Box 9 (SOX9)) from a transcription factor (TF) search website (http://www.cbrc.jp/ research/db/TFSEARCH.html). Then, we screened the effect of candidates on osteoblastic marker genes including $R U N X 2$, integrin binding sialoprotein (IBSP), and bone gamma-carboxyglutamic acid-containing protein (BGLAP) by measuring mRNA expression levels by real-time PCR. Total RNA was prepared from the normal cell line Nthy-Ori 3-1 and PTC cell lines TPC1 and BHP10-3, which were cultured in osteogenesis differentiation media for 0, 3, or 8 days; RNA from these cells was used as a template for real-time PCR (Fig. 1a). Based these data, HOXA9, for which expression changed over time with RUNX2, was selected as a primary candidate.

Subsequently, we cloned the RUNX2 P1 promoter and performed luciferase reporter assays to investigate the regulatory interaction between RUNX2 and HOXA9. The promoter activity of RUNX2 P1 was increased with the addition of HOXA9 (Fig. 1b). Next, we performed chromatin immunoprecipitation (ChIP) assays to assess the binding of HOXA9 to the promoter of RUNX2 using both normal Nthy-Ori 3-1 cells and TPC1 and BHP10-3 PTC lines, with an anti-HOXA9 antibody. Consistent with luciferase assay data, the binding of HOXA9 to the RUNX2 promoter improved in a dose-dependent manner (Fig. 1c). We also confirmed that RUNX2 gene expression was upregulated or downregulated depending on HOXA9 expression. These results suggest that HOXA9 regulates RUNX2 by binding its promoter in two types of thyroid cell lines, specifically control and PTC (Figs $1 \mathrm{~d}-\mathrm{f}$ and S1).

HOXA9 mediates the calcification of thyroid cells. To assess whether HOXA9 is involved in the process of calcification, alkaline phosphatase (ALP) staining was performed at 3, 5, and 7 days and Alizarin red S (ARS) staining was detected at 7-14 days. ALP activity was significantly enhanced in HOXA9-overexpressing Nthy-Ori 3-1 and TPC1 cells lines. In contrast, ALP activities were significantly reduced by the knockdown of this gene in TPC1 and BHP10-3 cell lines (Fig. 2a,b). Moreover, mineralization status was increased in HOXA9-overexpressing Nthy-Ori 3-1 and TPC1, but was attenuated in all HOXA9-knockdown groups (Fig. 2a,c). These data suggest that HOXA9 is involved in the process of thyroid calcification

HOXA9 is associated with thyroid cell migration and invasion. To assess Nthy-Ori 3-1, TPC1, and BHP10-3 cell migration and invasion, wound healing and invasion assays were performed. HOXA9-overexpressing cells migrated more than control cells, whereas in the normal Nthy-Ori 3-1 cell line, HOXA9 knockdown suppressed migration; however, this difference was not significant. In PTC cells, wound healing ability was increased marginally in HOXA9-overexpressing cells compared to that in control cells and was decreased in HOXA9-knockdown cells (Fig. 3a,b). Furthermore, cell invasion was enhanced in HOXA9-overexpressing groups and reduced in HOXA9-knockdown groups (Fig. 3c,d). These data indicate that HOXA9 can mediate migration and invasion in two types of thyroid cells.

HOXA9 enhances PTC calcification and tumour invasion directly or indirectly via RUNX2. To determine whether the HOXA9-mediated effect on enhanced calcification and carcinogenesis is associated with RUNX2, we utilized a RUNX2-knockdown system and HOXA9-overexpression system with the normal cell line Nthy-Ori 3-1 and PTC cell lines TPC1 and BHP10-3 (Figs 4a and S2).

Next, we performed mineralization assays, as previously described, on these cells. ALP activities were significantly decreased in all RUNX2-knockdown cells (Fig. 4b,c). Further, mineralization was also suppressed in all RUNX2-downregulated groups (Fig. 4b,d). ALP activity in Nthy-Ori 3-1 and BHP10-3 cells was marginally enhanced with RUNX2 knockdown and HOXA9 overexpression compared to that in RUNX2-knockdown control cells, and this difference was statistically significant $(p<0.05$ or $p<0.005$; Fig. $4 c)$.

Subsequently, wound healing and transwell assays were performed. Similar to the results of mineralization assays, migration was considerably decreased in RUNX2-knockdown cells compared to that in controls (Fig. 5a,c). Likewise, RUNX2 knockdown also suppressed invasion in the three thyroid cell lines. Additionally, invasion was enhanced in the groups of RUNX2-knockdown cells with HOXA9 overexpression compared to that in respective RUNX2-knockdown control cells (Fig. 5b,d).

These data suggest that HOXA9, as a positive regulator of RUNX2, can enhance calcification and tumour migration and invasion in PTC, dependent and independent of RUNX2.

\section{Discussion}

In this study, we screened upstream mediators of RUNX2, and found one candidate, HOXA9, which regulates the expression of this gene in thyroid cell lines. Overexpression of HOXA9 was found to enhance ALP activity and mineralization, as well as in vitro tumour cell migration and invasion, whereas downregulation of this marker inhibited these processes. Moreover, cells exhibited enhanced migration and invasion in RUNX2-knockdown 
a
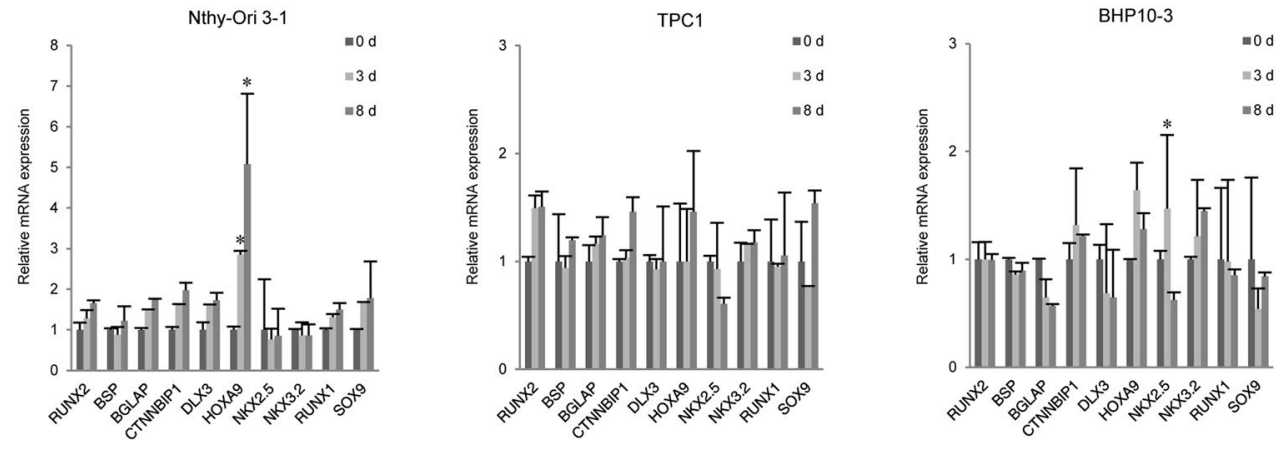

b
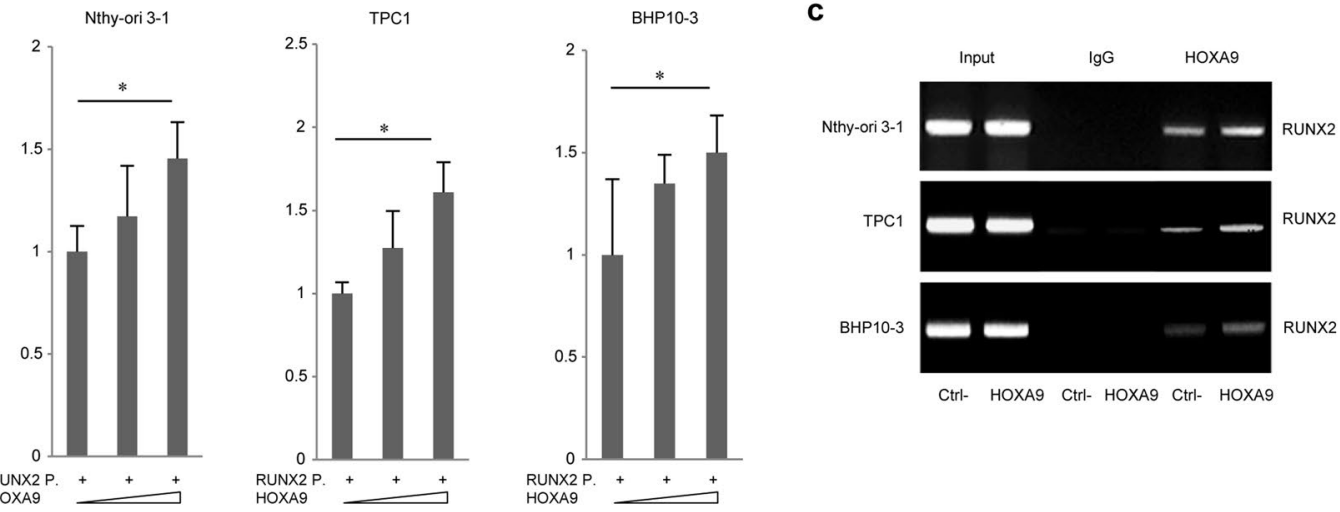

d

Nthy-ori 3-1
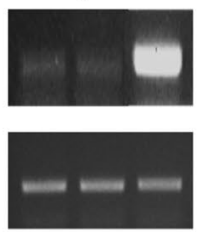

trl- ShHOXA9 HOXAG
TPC1
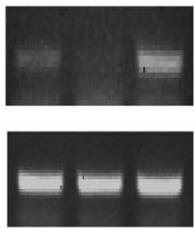

Ctrl- ShHOXA9HOXA9

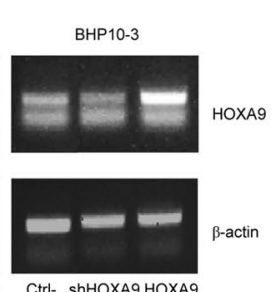

f

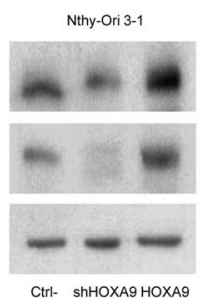

TPC1

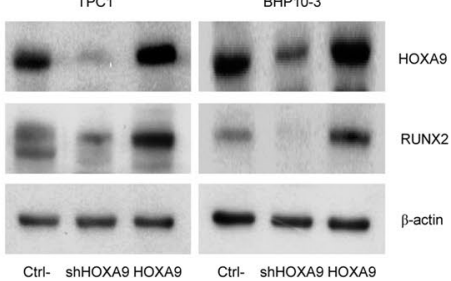

TPC1
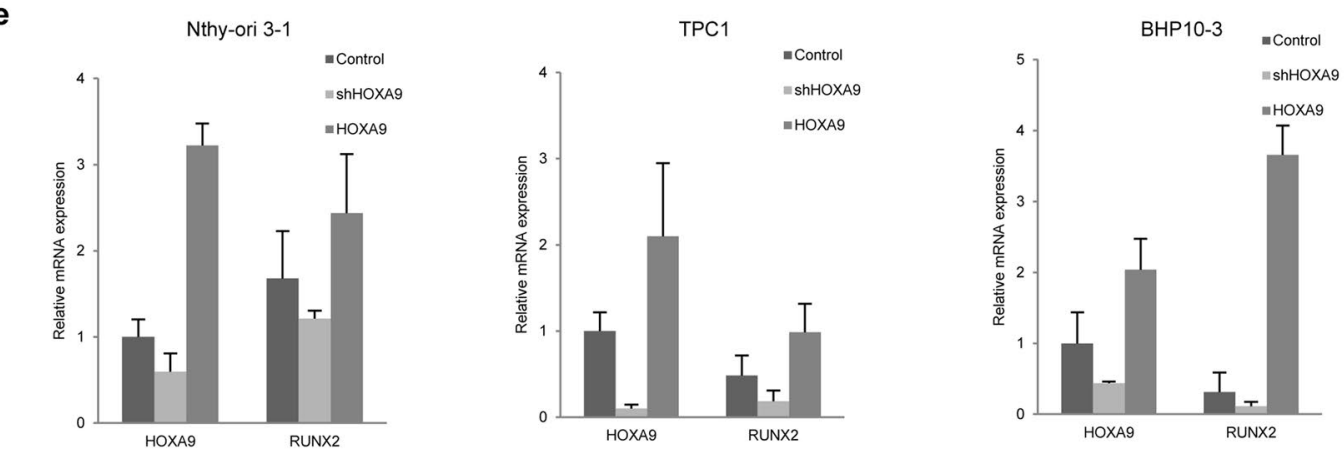

Figure 1. Expression of HOXA9 and RUNX2 in thyroid cells. (a) The expression of several selected candidate genes (RUNX2-upstream genes) in thyroid cell lines cultured in osteogenesis medium for 0,3 , and 8 days, as well as HOXA9 expression, was evaluated by real-time PCR. (b,c) The RUNX2 promoter (P) was cloned and plasmid DNA encoding HOXA9 was transfected into thyroid cells; HOXA9-binding activity and ability at the RUNX2 promoter region was assessed by luciferase reporter assays and chromatin immunoprecipitation (ChIP) assays. (d) HOXA9 was knocked down or overexpressed in two types of thyroid cell lines. The RNA expression of HOXA9 was assessed by qRT-PCR. (e,f) Alterations to the RNA and protein expression of RUNX2 depending on HOXA9 levels. Error bars represent standard deviation $(\mathrm{n}=3$ biological replicates). $* p<0.05$. The grouping of blots cropped from different parts of the same gel. The full-length blots are included in a Supplementary Information file (Supplementary Fig. S1).

cells with HOXA9 overexpression compared to those in RUNX2-knockdown control cells. This suggests that HOXA9 could be linked to the calcification and tumour invasion of PTC, which is both independent and dependent of RUNX2. 
a
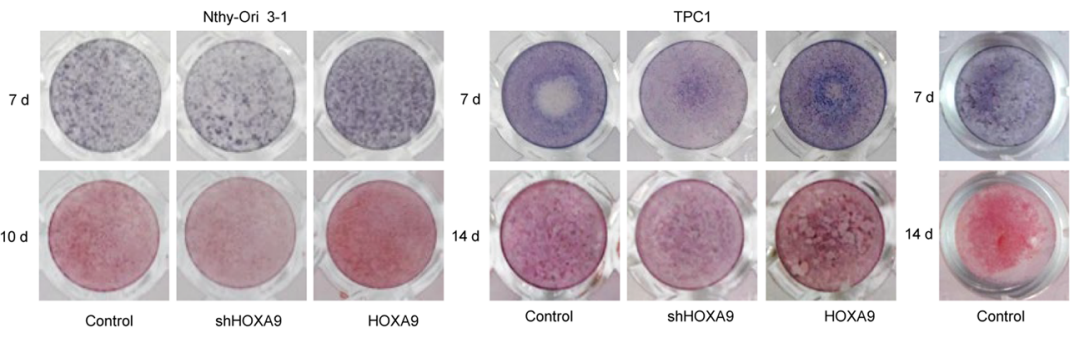

BHP10-3

b
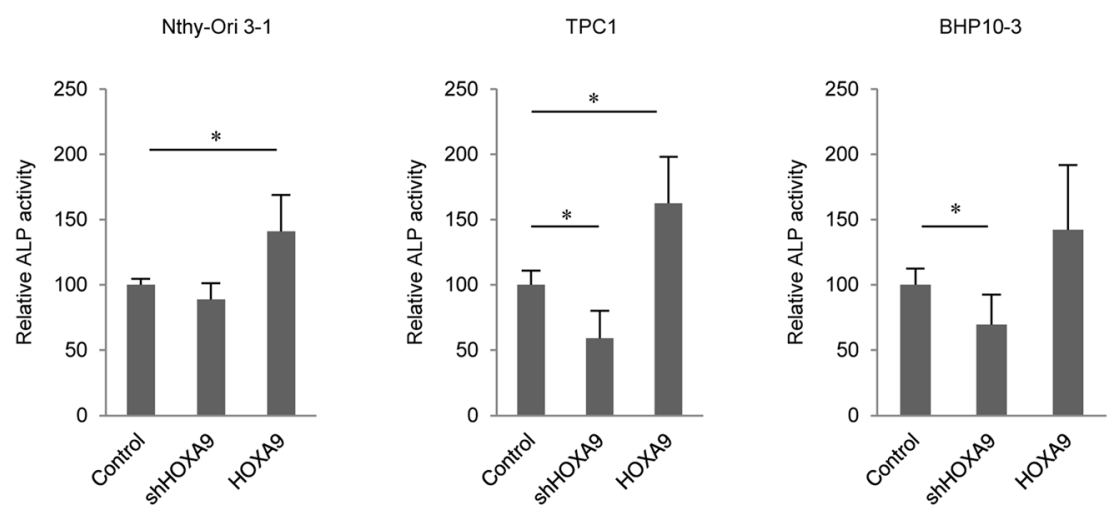

C
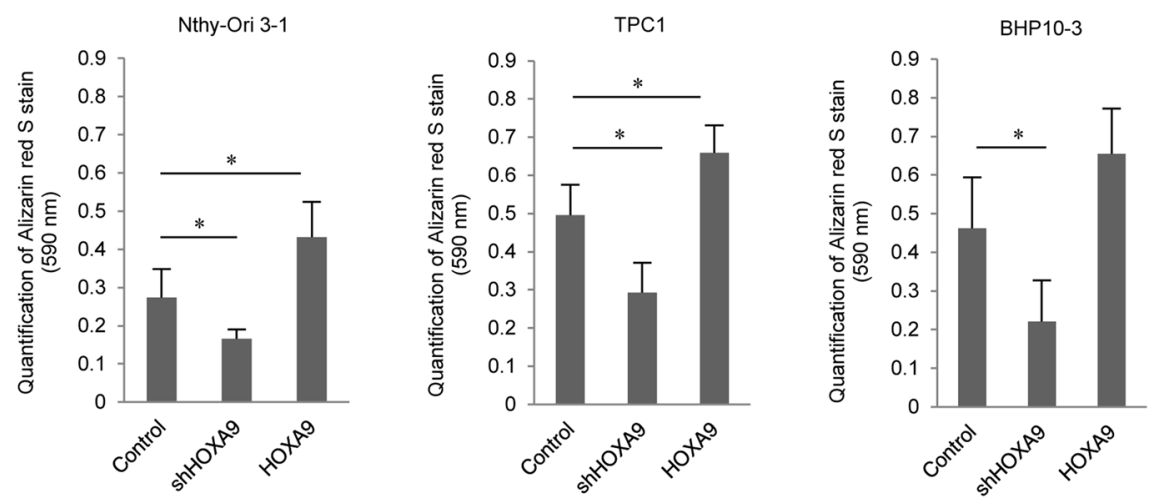

Figure 2. Effect of HOXA9 on osteoblast differentiation. (a) Alkaline phosphatase (ALP) staining (upper layer) was performed after 7 days and Alizarin red S staining was carried out on the $10^{\text {th }}$ day using Nthy-Ori 3-1 cells and on the $14^{\text {th }}$ day using papillary thyroid carcinoma (PTC) cells (TPC1 and BHP10-3). (b) ALP activity was measured at $405 \mathrm{~nm}$ using alkaline phosphatase yellow (pNPP) liquid substrate system with control, shHOXA9, and HOXA9-overexpressing cells for the two types of thyroid cells. (c) Alizarin red S-stained cells were extracted using cetylpyridinium chloride, and the mineralization level was quantified by measuring absorbance at $562 \mathrm{~nm}$. Error bars represent standard deviation $\left(\mathrm{n}=3\right.$ biological replicates). ${ }^{*} p<0.05$.

RUNX2 is the master regulator in osteoblast differentiation ${ }^{24-27}$. It was also reported to have a role in thyroid carcinogenesis. Specifically, levels of this marker were found to be significantly higher in larger PTC tumors ${ }^{25}$. Moreover, RUNX2 regulates cellular invasion in thyroid tumour cells ${ }^{26}$. However, there have been few reports regarding regulators of RUNX2 during thyroid calcification and carcinogenesis. Accordingly, we identified one candidate, HOXA9, and confirmed that it regulates the expression of RUNX2 in thyroid cell lines. Homeobox (HOX) family genes encode a class of transcription factors that regulate the expression of numerous genes, control cell growth, and drive specific tissue differentiation ${ }^{28}$. Further, the expression of HOX family genes is often dysregulated in tumours ${ }^{22}$. HOXA9, as a transcription factor, is most commonly altered in solid tumours ${ }^{23}$. To demonstrate the function of HOXA9 in PTC calcification, we performed ALP and ARS staining assays. ALP activity and calcification were enhanced in HOXA9-overexpressing Nthy-Ori 3-1 and TPC1 cells and reduced in HOXA9-knockdown TPC1 and BHP10-3 cells. These results indicated that calcification could be regulated by HOXA9 in thyroid cell lines.

Many studies have established the function of HOXA9 in cancer cells. There are some reports indicating that HOXA9 knockdown can significantly decrease colony formation, invasion, and migration in colorectal cancer cells ${ }^{29-31}$. However, some others reported that HOXA9 inhibits migration and that the hypermethylation of HOXA9 is especially apparent in the early stages of lung cancer ${ }^{32-34}$. Moreover, low expression of this marker was observed in cervical cancer cells and proliferation and migration were suppressed when HOXA9 expression was 
a

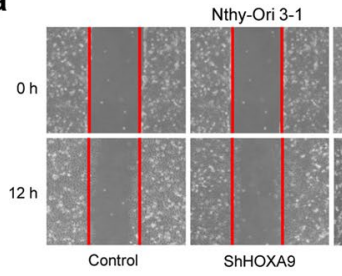

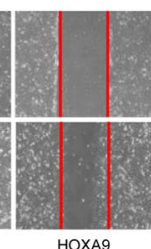

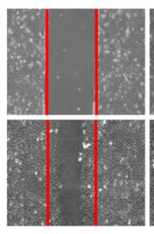

Control
TPC1

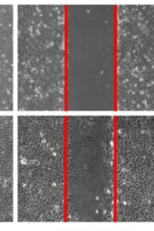

ShHOXA9

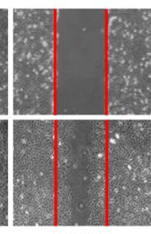

HOXA9

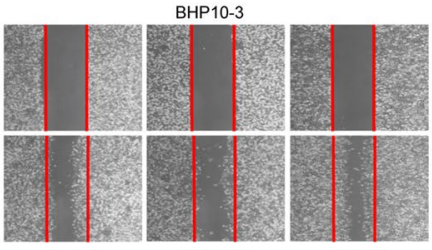

Control

ShHOXA9

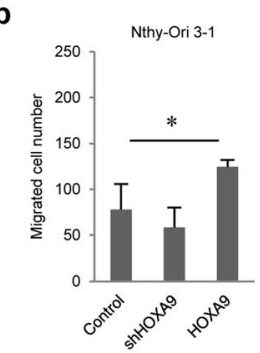

C

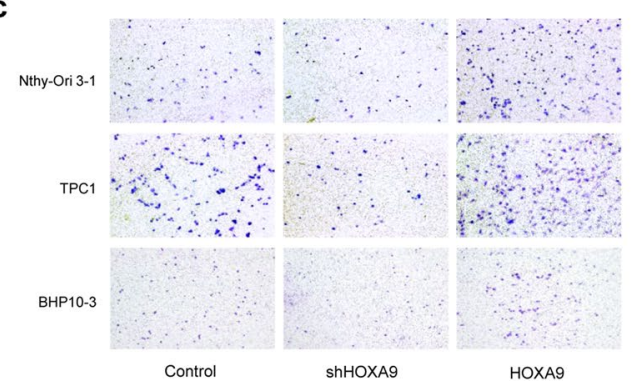

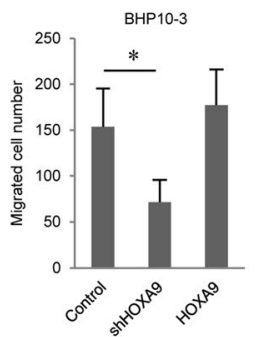

d

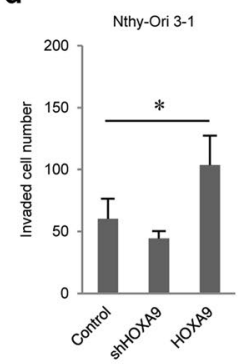

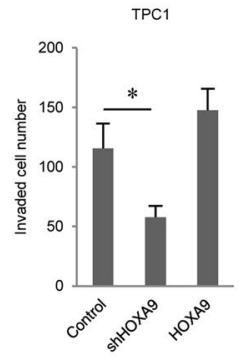

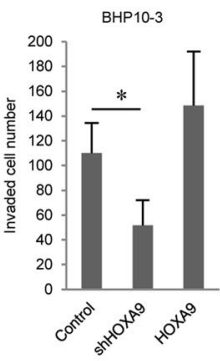

Figure 3. Migration and invasion ability of thyroid cells based on HOXA9 expression. (a) Thyroid cells were seeded in 6 -well plates $\left(6 \times 10^{4}\right.$ cells/well $)$ with $2 \mathrm{ml}$ of complete growth media. After $24 \mathrm{~h}$, wound-healing assays were performed, and the wells were photographed every $12 \mathrm{~h}$ to monitor wound closure. (c) Thyroid cells were transferred to the top of Matrigel-coated chambers with $3 \times 10^{4}$ cells/well in serum-free media. After $24 \mathrm{~h}$ of incubation, the invaded cells were fixed with methanol and stained with $0.5 \%$ crystal violet. (b,d) The numbers of migrating cells into the wound and invading cells were counted under a microscope. Experiments were repeated three times. Error bars represent standard deviation $(\mathrm{n}=3$ biological replicates). $* p<0.05$.

restored in these cells ${ }^{35}$. This protein was also found to restrict cell growth, survival, and invasion in T4-2 breast cancer cells ${ }^{36}$. In contrast, it was determined that HOXA9 promotes the viability and aggressiveness of glioblastoma cells ${ }^{37}$. Further, in ovarian cancer, this factor was shown to induce peritoneal macrophages to obtain an M2 tumour-promoting phenotype ${ }^{38}$. Likewise, HOXA9 encourages epithelial ovarian cancer growth in mouse xenograft models and supports the generation of a microenvironment for tumour growth ${ }^{39}$. However, there have been no reports regarding the role of HOXA9 in thyroid carcinoma. This study showed that the overexpression of HOXA9 significantly enhances in vitro normal cell migration and invasion and that tumour cell migration and invasion can be inhibited by downregulating HOXA9. Our results thus showed that HOXA9 could be related to thyroid cell migration an invasion.

As stated, HOXA9 is an upstream regulator of RUNX2. Therefore, we investigated whether its effect on enhanced calcification and carcinogenesis occurs through RUNX2. Thus, we downregulated RUNX2 in two types of thyroid cell lines with or without HOXA9 overexpression. Our results indicated that ALP activity, mineralization behaviour, and cell migration and invasion capacity were all significantly decreased upon RUNX2 downregulation. These results were similar to a previous report showing that the downregulation of RUNX2 can impair migration and invasion in thyroid tumour cells ${ }^{26}$. However, all of these activities were increased with HOXA9 overexpression, as compared to those in the RUNX2-knockdown only groups. These results showed that HOXA9 might enhance the calcification and tumour invasion of PTC not only via RUNX2, but also independently of this marker.

In conclusion, HOXA9, a positive regulator of RUNX2, can enhance calcification and cancer migration and invasion ability in PTC, dependent and independent of RUNX2. Our data improve the understanding of the molecular mechanisms of microcalcification and tumorigenesis in PTC and might lead to the development of novel diagnostic or prognostic biomarkers for this disease.

\section{Methods}

Cell culture. The human normal thyroid cell line Nthy-Ori 3-1 and PTC cell line BHP10-3 were maintained in complete Roswell Park Memorial Institute 1640 medium (RPMI1640; Welgene, Gyeongsan, Korea) with 10\% foetal bovine serum (FBS; Gibco BRL) and 1\% antibiotic-antimycotic (Gibco, New York, NY); the other PTC cell line TPC1 was grown in Dulbecco's Modified Eagle Medium/Nutrient Mixture F-12, 1:1 Mixture (DMEM/F12; 
a

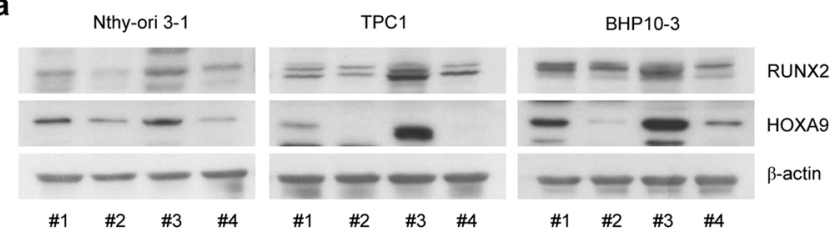

b

Nhy-Ori 3-1

TPC1
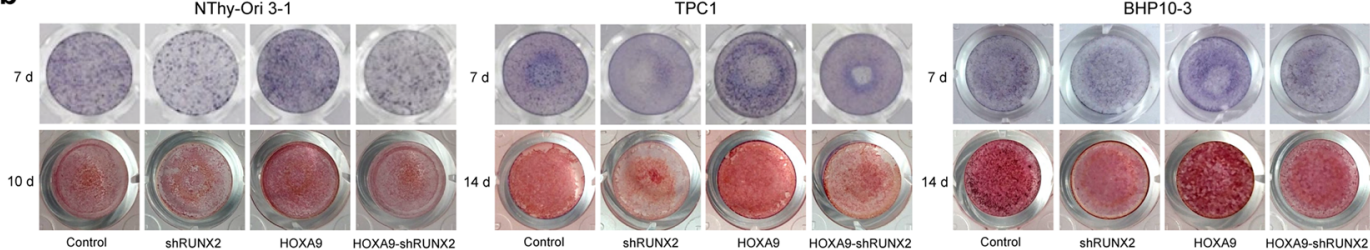

C
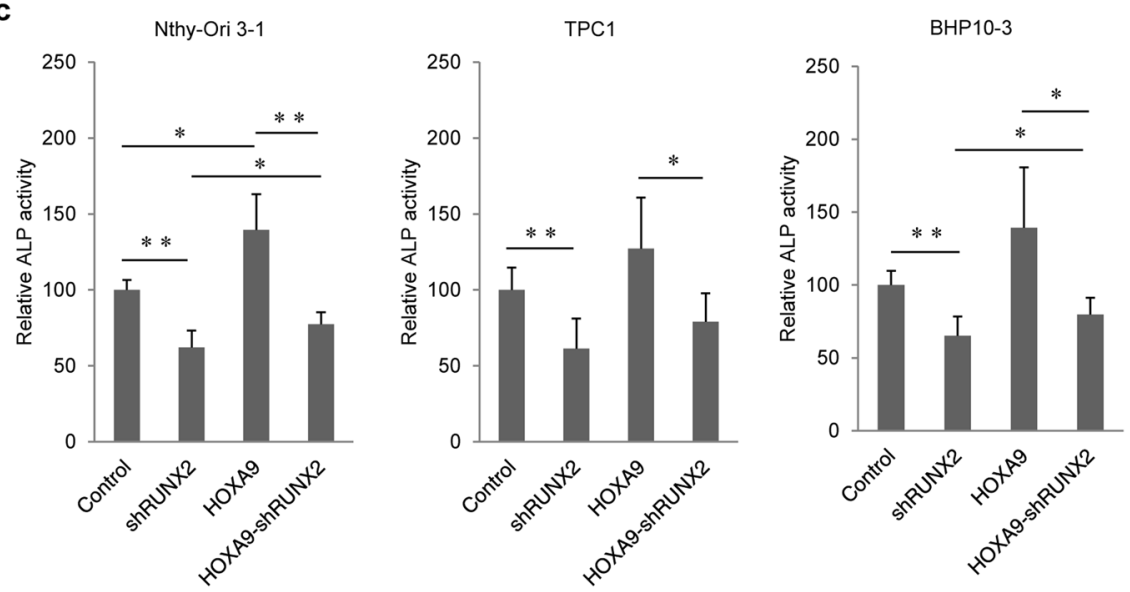

d
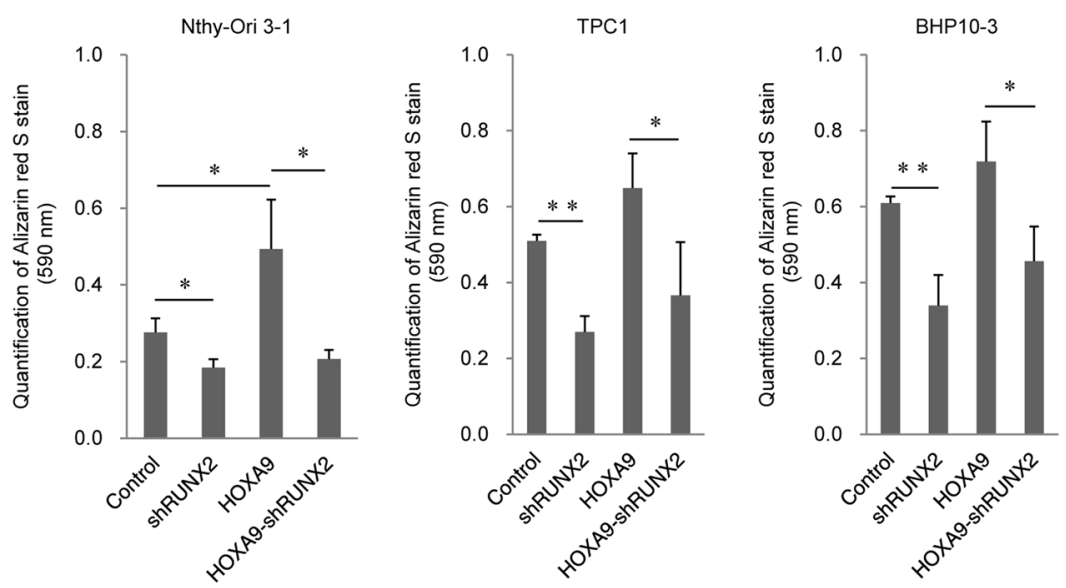

Figure 4. Effect of HOXA9 overexpression and shRUNX2 on osteoblast differentiation. (a) RUNX2 was knocked down in three control and HOXA9-overexpressing cell lines. Protein expression was assessed by western blotting (\#1: Control, \#2: shRUNX2, \#3: HOXA9-overexpressing, \#4: HOXA9-overexpressing/ shRUNX2). The grouping of blots cropped from different parts of the same gel. The full-length blots are included in a Supplementary Information file (Supplementary Fig. S2). (b) Alkaline phosphatase (ALP) staining (upper layer) on the $7^{\text {th }}$ day and Alizarin red S staining (lower layer) on the $10^{\text {th }}$ day for Nthy-Ori 3-1 cells and on the $14^{\text {th }}$ day for papillary thyroid carcinoma (PTC) cells (TPC1 and BHP10-3). (c,d) Quantitative data for ALP and ARS staining in thyroid cells with control, shRUNX2, HOXA9-overexpressing, and HOXA9overexpressing/shRUNX2 conditions. Error bars represent standard deviation ( $\mathrm{n}=3$ biological replicates). $* p<0.05, * * p<0.005$.

Welgene, Gyeongsan, Korea) with 10\% FBS and 1\% antibiotic-antimycotic. For osteoblast differentiation, cells were plated at $1.0 \times 10^{4}$ cells/well in 48 -well plates and used for calcification assays and for PCR; $2.0 \times 10^{4}$ cells were seeded in a $60-\mathrm{mm}$ plate and cells were induced by osteogenic medium after $24 \mathrm{~h}$ (for cell adherence; day 0 ). Medium was changed every 2 days. All cells were incubated in a humidified atmosphere of $5 \% \mathrm{CO}_{2}$ at $37^{\circ} \mathrm{C}$. 
a
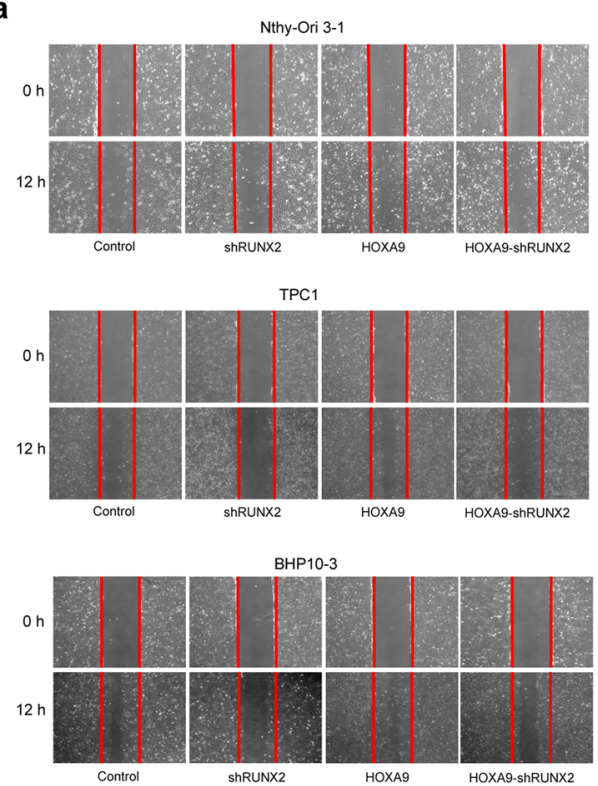

b

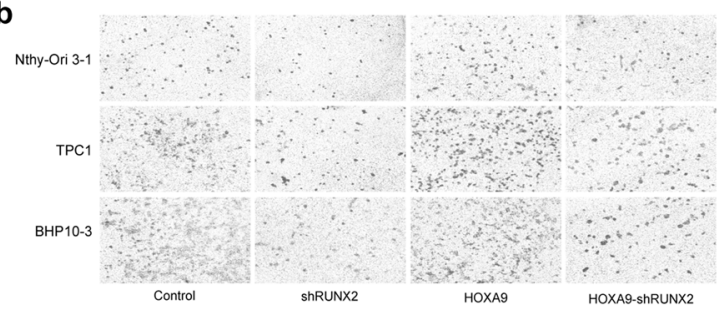

C

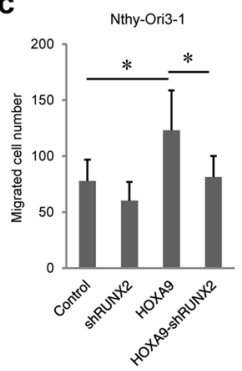

d

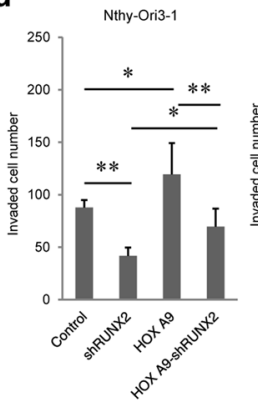

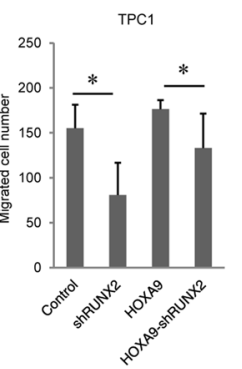
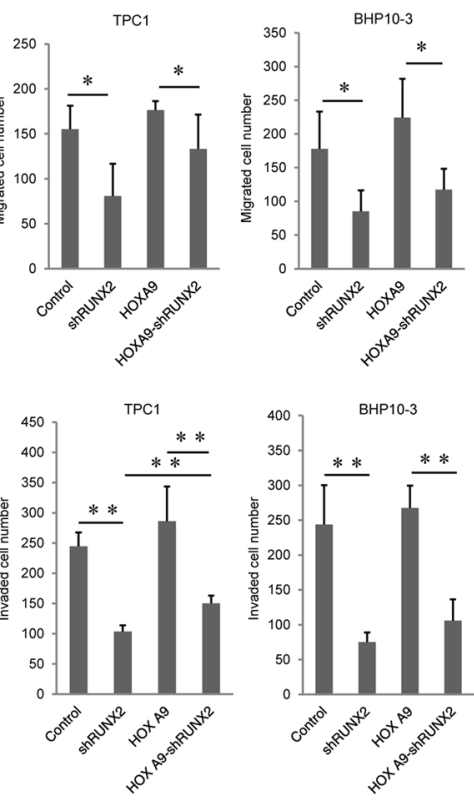

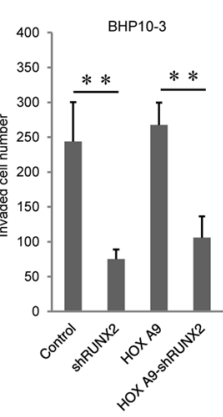

Figure 5. Migration and invasion ability based on HOXA9 and RUNX2 expression in thyroid cells. (a) Migration and invasion ability were demonstrated by wound-healing and transwell assays. Cells were seeded in 6 -well plates $\left(6 \times 10^{4}\right.$ cells/well), and after $24 \mathrm{~h}$, wound-healing assays were performed and the wells were photographed every $12 \mathrm{~h}$ to monitor wound closure. (b) Thyroid cells were transferred to the top of Matrigelcoated chambers with $3 \times 10^{4}$ cells/well in serum-free media. After $24 \mathrm{~h}$ of incubation, the invaded cells were fixed with methanol and stained with $0.5 \%$ crystal violet. (c) The number of cells that migrated into the wound. (d) The number of cells that invaded through the filter. The numbers of migrated cells into the wound and invaded cells were counted under a microscope. Experiments were repeated three times. Error bars represent standard deviation $(\mathrm{n}=3$ biological replicates). $* p<0.05, * * p<0.005$.

Candidate regulators of RUNX2. To discover a novel gene that regulates RUNX2, we used the transcription factor search website http://www.cbrc.jp/research/db/TFSEARCH.html with the 3000-bp RUNX2 promoter and identified putative upstream markers. CTNNBIP1, DLX3, HOXA9, NKX2-5, NKX3-2, RUNX1, and SOX9, with scores greater than 95.00, were selected as candidates.

Quantitative reverse-transcription PCR (qRT-PCR) and real-time PCR. To determine mRNA expression levels of the bone metabolic markers RUNX2, IBSP, and BGLAP (Osteocalcin) and candidate genes including CTNNBIP1, DLX3, HOXA9, NKX2-5, NKX3-2, RUNX1, and SOX9, qRT-PCR and real-time PCR were performed. To normalize the efficiency of real-time RT-PCR reactions, human $\beta$-actin was used as a standard. Total RNA was extracted from cultured cells in osteogenesis medium at 0,3 , and 8 days cells using TRIzol reagent (Invitrogen, Carlsbad, USA) following the manufacturer's instructions and quantified using a spectrophotometer (Beckman Coulter, Brea, USA). Extracted RNA was subsequently reverse transcribed at $42^{\circ} \mathrm{C}$ for $1 \mathrm{~h}$ using a premix kit with oligo-dT as a primer (iNtRON Biotechnology, Seongnam, Korea). Next, 27-34 cycles of qRT-PCR were performed using a Maxime PCR PreMix kit (i-StarTaq; iNtRON Biotechnology, Seongnam, Korea). All real-time PCR measurements were performed using the ABI Prism 7000 Sequence Detection System (Applied Biosystems; Foster City, USA). All PCR amplifications (25 cycles) were performed in a total volume of $25 \mu \mathrm{l}$ containing 150 ng cDNA using the SYBR Green I qPCR kit (TaKaRa, Shiga, Japan) according to the manufacturer's recommendations. The amplification parameters were in accordance with the manufacturer's recommendations. By normalizing to $\beta$-actin, relative quantification of gene expression was performed using the comparative threshold (Ct) method as described by the manufacturer (Applied Biosystems). The values were expressed as fold-change relative to control levels. Relative gene expression was displayed as $2-\Delta \mathrm{Ct}(\Delta \mathrm{Ct}=\mathrm{Ct}$ target gene $-\mathrm{Ct} \beta$-actin). Fold-change was calculated as $2-\Delta \Delta \mathrm{Ct}(\Delta \Delta \mathrm{Ct}=\Delta \mathrm{Ct}$ control $-\mathrm{Ct}$ treatment $)$. PCR primers are described in Table 1. 


\begin{tabular}{|c|c|}
\hline Gene & Primer (qRT-PCR) \\
\hline \multirow{2}{*}{$\beta$-actin } & 5'-CCTAAAAGCCACCCСACTTC-3' \\
\hline & 5'-AGGGAGACCAAAAGCCTTCA-3' \\
\hline \multirow{2}{*}{ RUNX2 } & $5^{\prime}$-TTACTTACACCCCGCCAGTC-3' \\
\hline & 5'-TATGGAGTGCTGCTGGTCTG-3' \\
\hline \multirow{2}{*}{ HOXA9 } & 5'-CCACGCTTGACACTCACACT-3' \\
\hline & 5'-CAGTTCCAGGGTCTGGTGTT-3' \\
\hline Gene & Primer (real-time PCR) \\
\hline \multirow{2}{*}{$\beta$-actin } & 5'-CAAGATCAACCGGGAAAAGA-3' \\
\hline & 5'-CTGAGGCATAGAGGGACAGC-3' $^{\prime}$ \\
\hline \multirow{2}{*}{ RUNX2 } & 5'-TTACTTACACCCCGCCAGTC-3' \\
\hline & 5'-CACTCTGGCTTTGGGAAGAG-3' \\
\hline \multirow{2}{*}{ Bsp } & 5'-AACCTACAACCCCACCACAA-3' \\
\hline & 5'-CGTACTCCCCCTCGTATTCA-3' \\
\hline \multirow{2}{*}{ BGLAP } & 5'-GACTGTGACGAGTTGGCTGA-3' \\
\hline & 5'-CTGGAGAGGAGCAGAACTGG-3' \\
\hline \multirow{2}{*}{ CTNNBIP1 } & 5'-ACCTTTCCCATCATCGTGAG-3' \\
\hline & 5'-AATCCACTGGTGAACCAAGC-3' \\
\hline \multirow{2}{*}{ DLX3 } & 5'-AGGCCTAGTTCCTCCTGAGC-3' \\
\hline & 5'-CCTCGTCATGATGTCCACTG-3' \\
\hline \multirow{2}{*}{ HOXA9 } & 5'-CAATAACCCAGCAGCCAACT-3' \\
\hline & 5'-CAGTTCCAGGGTCTGGTGTT-3' \\
\hline \multirow{2}{*}{ NKX2-5 } & 5'-GTCAAGCCGCTCTTACCAAG-3' \\
\hline & 5'-TTGTCCGCCTCTGTCTTCTC-3' \\
\hline \multirow{2}{*}{ NKX3-2 } & 5'-GACGCAGGTGAAAATCTGGT-3' \\
\hline & 5'-ACCTTTACGGCCACCTTCTT-3' \\
\hline \multirow{2}{*}{ Runx1 } & 5'-GGCTGGCAATGATGAAAACT-3' \\
\hline & 5'-CCGACAAACCTGAGGTCATT- ${ }^{\prime}{ }^{\prime}$ \\
\hline
\end{tabular}

Table 1. Primers for qRT-PCR and real-time PCR.

Luciferase reporter and ChIP assays. To analyse RUNX2 promoter activity, Nthy-Ori 3-1, TPC1, and BHP10-3 cells were plated on 24 -well plates at a density of $2 \times 10^{4}$ cells/well 1 day before transfection. Plasmid DNA was mixed with TransIT-2020 (Mirus, Madison, WI) and transfected into the cells following the manufacturer's protocol. After $48 \mathrm{~h}$, cells were washed twice with $1 \times \mathrm{PBS}$ and then lysed in reporter lysis buffer (Promega, Madison, WI). Cell extracts were subjected to assays using a luciferase assay system (Promega, Madison, WI) according to the manufacturer's instructions. Luciferase activity was measured in triplicate, averaged, and then normalized to $\beta$-galactosidase activity using o-nitrophenyl- $\beta$-D-galactopyranoside (Sigma-Aldrich) as a substrate.

ChIP assays were performed with a ChIP kit (Upstate Biotechnology, Lake Placid. NY), with modified instructions from the manufacturer, using antibodies against HOXA9 (Proteintech, Rosemont, USA) or control IgG (Santa Cruz Biotechnology, Santa Cruz, CA). The precipitated DNA was subjected to PCR amplification with specific primers for the RUNX2 P1 promoter region, which contains HOXA9-binding sites. The following primers were used for PCR: RUNX2 P1 sense, 5'-GCAAAAAGGCAGAGGTTGAG-3'; RUNX2 P1 antisense, 5'-CCCCCTTGCTCTTTCTCTCT-3'.

Plasmids, lentivirus packaging, and stable cell lines. We purchased full-length HOXA9wt, pOTB7-HOXA9 from the Korea Human Gene Bank, Medical Genomics Research Center, KRIBB, Korea. HOXA9 was subcloned into the pcDNA3.1 (+) vector (Invitrogen) by ligating NheI-XhoI fragments to generate the pcDNA-HOXA9 expression construct. pcDNA-HOXA9 was confirmed by DNA sequencing. Stable cell lines were established by transfecting HOXA9 plasmids into thyroid cells and selecting with G418 at a concentration of $400 \mu \mathrm{g} / \mathrm{ml}$.

Lentivirus harbouring HOXA9 or RUNX2 or vector controls were designed and packaged by Merck (Darmstadt, Germany). Lentiviruses were packaged in HEK-293T cells and collected from the medium supernatant. Stable cell lines were established by infecting thyroid cells with HOXA9- or RUNX2-expressing lentiviruses and selecting with puromycin at a concentration of $10 \mu \mathrm{g} / \mathrm{ml}$.

Western blotting. Cell lysates were extracted in $0.1 \mathrm{M} \mathrm{NaCl}, 0.01 \mathrm{M}$ tris- $\mathrm{HCl}(\mathrm{pH} 7.6), 1 \mathrm{mM}$ EDTA (pH $8.0), 1 \mathrm{mg} / \mathrm{ml}$ aprotinin, and $100 \mathrm{mg} / \mathrm{ml}$ PMSF; protein concentrations were determined by a Bio-Rad protein assay. Protein $(50 \mu \mathrm{g})$ was boiled at $95^{\circ} \mathrm{C}$ in sodium dodecyl sulphate (SDS) sample buffer for 5 min, electrophoresed on $10 \%$ or $12 \%$ SDS-PAGE gels, and transferred to polyvinyldifluoridine membranes. Then, blots were incubated overnight at $4^{\circ} \mathrm{C}$ with anti-HOXA9 (Abcam, Cambridge, UK, \#ab191178; 1:1000), anti-RUNX2 (Abcam, Cambridge, UK, \#ab23981; 1:1000), or anti- $\beta$-actin (Bethyl, Montgomery, Texas, USA, \#A300-491A; 
1:8000) antibodies. Membranes incubated with anti-goat or anti-rabbit secondary antibody (Santa Cruz Biotechnology; 1:5000) at room temperature for $60 \mathrm{~min}$. Protein band signals were visualized using an ECF western blotting kit (Amersham Biosciences, Piscataway, NJ, USA) and detected with an LAS3000 luminescent image analyzer (Fuji Photo Film).

ALP assays and ARS staining. For ALP staining, cultured cells were fixed in $10 \%$ formalin for $10 \mathrm{~min}$, permeabilized for $30 \mathrm{~min}$ with $0.1 \%$ triton-100 in $1 \times$ PBS, and treated with nitro blue tetrazolium and 5-bromo4-chloro-3-indolyl phosphate for 10-30 min. To measure calcium deposition in the extracellular matrix, $200 \mu \mathrm{l}$ of extraction solution was added and samples were incubated overnight at $4{ }^{\circ} \mathrm{C}$. ALP activity was measured in total cell lysates after homogenization in a buffer containing $1 \mathrm{mM}$ tris- $\mathrm{HCl}(\mathrm{pH} 8.8), 0.5 \%$ triton X-100, $10 \mathrm{mM} \mathrm{Mg}^{2+}$, and $5 \mathrm{mM}$ p-nitrophenyl phosphate as substrates. The absorbance was read at $405 \mathrm{~nm}$ (BioTek, USA).

For ARS staining, cultured cells were fixed in $70 \%$ ethyl alcohol for $1 \mathrm{~h}$. After washes with $1 \times$ PBS, cells were stained with $40 \mathrm{mM}$ ARS solution ( $\mathrm{pH} \mathrm{4.2)} \mathrm{for} 10 \mathrm{~min}$ to stain calcium deposits. Cells were then washed five times with distilled water followed by $1 \times$ PBS for $15 \mathrm{~min}$ and non-specific stained cells were removed. To quantify the degree of mineralization, cells were extracted using $10 \%(\mathrm{w} / \mathrm{v})$ cetylpyridinium chloride in $10 \mathrm{mM}$ sodium phosphate ( $\mathrm{pH} 7.0$ ). The concentration was evaluated by measuring the absorbance at $562 \mathrm{~nm}$ with a multiplate reader using an Alizarin red S standard curve in the same solution. All values are expressed as fold-change relative to the control.

Wound healing assays. Thyroid cells were seeded in 6 -well plates $\left(6 \times 10^{4}\right.$ cells/well $)$ with $2 \mathrm{ml}$ of complete RPMI1640 or DMEM/F-12. At $24 \mathrm{~h}$, the monolayers were mechanically disrupted with a pipette tip to produce a clean uniform scratch. The assay was performed three times and wells were photographed every $12 \mathrm{~h}$ to monitor the closing of the wound. The number of migrating cells into the wound was counted under a microscope. Experiments were repeated three times.

Invasion assays. Matrigel was coated on 24-well chambers (Corning, NY, USA, \#3415), and thyroid cells were transferred at a density of $3 \times 10^{4}$ on top of the Matrigel-coated chambers in serum-free media after drying the Matrigel. After $24 \mathrm{~h}$ of incubation, non-invading cells were removed by rinsing, and invaded cells were fixed with ice-cold $100 \%$ methanol and stained with $0.5 \%$ crystal violet. The number of invading cells was manually counted in four randomly chosen fields under a microscope and images were captured at $\times 100$ magnification. Experiments were repeated three times.

Statistical analysis. The statistical significance of differences was assessed by performing a Student's t-test. $P<0.05$ was considered significant. Results are expressed as the mean $\pm \mathrm{SEM}$.

\section{Data Availability}

The datasets used and/or analysed during the current study are available from the corresponding author upon reasonable request.

\section{References}

1. Sipos, J. A. \& Mazzaferri, E. L. Thyroid cancer epidemiology and prognostic variables. Clin Oncol (R Coll Radiol) 22, 395-404, https://doi.org/10.1016/j.clon.2010.05.004 (2010).

2. Lloyd, R. V. Endocrine Pathology:: Differential Diagnosis and Molecular Advances. (Springer Science \& Business Media, 2010).

3. DeLellis, R. A. Pathology and genetics of tumours of endocrine organs. Vol. 8 (IARC, 2004).

4. Carcangiu, M. L., Zampi, G., Pupi, A., Castagnoli, A. \& Rosai, J. Papillary carcinoma of the thyroid. A clinicopathologic study of 241 cases treated at the University of Florence, Italy. Cancer 55, 805-828 (1985).

5. Johannessen, J. V. \& Sobrinho-Simoes, M. The origin and significance of thyroid psammoma bodies. Lab Invest 43, 287-296 (1980),

6. Lacout, A., Chevenet, C., Thariat, J. \& Marcy, P. Y. Thyroid calcifications: a pictorial essay. J Clin Ultrasound 44, 245-251, https://doi. org/10.1002/jcu.22345 (2016).

7. Cotran, R. S., Kumar, V., Collins, T. \& Robbins, S. L. Robbins pathologic basis of disease. (1999).

8. Underwood, J. C. \& Cross, S. S. General and Systematic Pathology, International Edition E-Book: with STUDENT CONSULT Access. (Elsevier Health Sciences, 2009).

9. Das, D. K. Psammoma body: a product of dystrophic calcification or of a biologically active process that aims at limiting the growth and spread of tumor? Diagn Cytopathol 37, 534-541, https://doi.org/10.1002/dc.21081 (2009).

10. Harada, H. et al. Cbfa1 isoforms exert functional differences in osteoblast differentiation. The Journal of biological chemistry 274, 6972-6978 (1999).

11. Cohen, M. M. Jr. Perspectives on RUNX genes: an update. Am J Med Genet A 149A, 2629-2646, https://doi.org/10.1002/ ajmg.a.33021 (2009).

12. Zambotti, A., Makhluf, H., Shen, J. \& Ducy, P. Characterization of an osteoblast-specific enhancer element in the CBFA1 gene. The Journal of biological chemistry 277, 41497-41506, https://doi.org/10.1074/jbc.M204271200 (2002).

13. Pratap, J., Lian, J. B. \& Stein, G. S. Metastatic bone disease: role of transcription factors and future targets. Bone 48, 30-36, https:// doi.org/10.1016/j.bone.2010.05.035 (2011)

14. Wysokinski, D., Blasiak, J. \& Pawlowska, E. Role of RUNX2 in Breast Carcinogenesis. Int J Mol Sci 16, 20969-20993, https://doi. org/10.3390/ijms160920969 (2015).

15. Chang, C. H. et al. The prognostic significance of RUNX2 and miR-10a/10b and their inter-relationship in breast cancer. J Transl Med 12, 257, https://doi.org/10.1186/s12967-014-0257-3 (2014).

16. Trotter, T. N. et al. Myeloma cell-derived Runx2 promotes myeloma progression in bone. Blood 125, 3598-3608, https://doi. org/10.1182/blood-2014-12-613968 (2015).

17. Dalle Carbonare, L. et al. Runx2 mRNA expression in the tissue, serum, and circulating non-hematopoietic cells of patients with thyroid cancer. J Clin Endocrinol Metab 97, E1249-1256, https://doi.org/10.1210/jc.2011-2624 (2012).

18. Endo, T., Ohta, K. \& Kobayashi, T. Expression and function of Cbfa-1/Runx2 in thyroid papillary carcinoma cells. JClin Endocrinol Metab 93, 2409-2412, https://doi.org/10.1210/jc.2007-2805 (2008).

19. Niu, D. F. et al. Transcription factor Runx2 is a regulator of epithelial-mesenchymal transition and invasion in thyroid carcinomas. Lab Invest 92, 1181-1190, https://doi.org/10.1038/labinvest.2012.84 (2012). 
20. Morgan, R. \& El-Tanani, M. HOX Genes as Potential Markers of Circulating Tumour Cells. Current molecular medicine 16, 322-327 (2016).

21. Morgan, R., El-Tanani, M., Hunter, K. D., Harrington, K. J. \& Pandha, H. S. Targeting HOX/PBX dimers in cancer. Oncotarget 8 , 32322-32331, https://doi.org/10.18632/oncotarget.15971 (2017).

22. Samuel, S. \& Naora, H. Homeobox gene expression in cancer: insights from developmental regulation and deregulation. Eur J Cancer 41, 2428-2437, https://doi.org/10.1016/j.ejca.2005.08.014 (2005).

23. Bhatlekar, S., Fields, J. Z. \& Boman, B. M. HOX genes and their role in the development of human cancers. Journal of molecular medicine (Berlin, Germany) 92, 811-823, https://doi.org/10.1007/s00109-014-1181-y (2014).

24. Soltanoff, C. S., Yang, S., Chen, W. \& Li, Y. P. Signaling networks that control the lineage commitment and differentiation of bone cells. Critical reviews in eukaryotic gene expression 19, 1-46 (2009).

25. Gong, T., Wang, J., Qian, M. \& Zhou, Y. [Detection of Runx2 mRNA expression using relatively real-time RT-PCR in papillary thyroid carcinoma]. Lin chuang er bi yan hou tou jing wai ke za zhi=Journal of clinical otorhinolaryngology, head, and neck surgery 27, 193-195 (2013).

26. Sancisi, V. et al. Runx2 isoform I controls a panel of proinvasive genes driving aggressiveness of papillary thyroid carcinomas. J Clin Endocrinol Metab 97, E2006-2015, https://doi.org/10.1210/jc.2012-1903 (2012).

27. Pratap, J. et al. Cell growth regulatory role of Runx2 during proliferative expansion of preosteoblasts. Cancer research 63, 5357-5362 (2003).

28. Grier, D. G. et al. The pathophysiology of HOX genes and their role in cancer. The Journal of pathology 205, 154-171, https://doi. org/10.1002/path.1710 (2005).

29. Wang, X. et al. miR-133b suppresses metastasis by targeting HOXA9 in human colorectal cancer. Oncotarget 8, 63935-63948, https://doi.org/10.18632/oncotarget.19212 (2017).

30. Bhatlekar, S., Viswanathan, V., Fields, J. Z. \& Boman, B. M. Overexpression of HOXA4 and HOXA9 genes promotes self-renewal and contributes to colon cancer stem cell overpopulation. Journal of cellular physiology 233, 727-735, https://doi.org/10.1002/jcp.25981 (2018).

31. Gaspar, N. et al. MGMT-independent temozolomide resistance in pediatric glioblastoma cells associated with a PI3-kinasemediated HOX/stem cell gene signature. Cancer research 70, 9243-9252, https://doi.org/10.1158/0008-5472.can-10-1250 (2010).

32. Hwang, J. A. et al. HOXA9 inhibits migration of lung cancer cells and its hypermethylation is associated with recurrence in nonsmall cell lung cancer. Molecular carcinogenesis 54(Suppl 1), E72-80, https://doi.org/10.1002/mc.22180 (2015).

33. Hwang, S. H. et al. Detection of HOXA9 gene methylation in tumor tissues and induced sputum samples from primary lung cancer patients. Clinical chemistry and laboratory medicine 49, 699-704, https://doi.org/10.1515/cclm.2011.108 (2011).

34. Wrangle, J. et al. Functional identification of cancer-specific methylation of CDO1, HOXA9, and TAC1 for the diagnosis of lung cancer. Clinical cancer research: an official journal of the American Association for Cancer Research 20, 1856-1864, https://doi. org/10.1158/1078-0432.ccr-13-2109 (2014).

35. Alvarado-Ruiz, L. et al. HOXA9 is Underexpressed in Cervical Cancer Cells and its Restoration Decreases Proliferation, Migration and Expression of Epithelial-to-Mesenchymal Transition Genes. Asian Pacific journal of cancer prevention: APJCP 17, 1037-1047 (2016).

36. Gilbert, P. M. et al. HOXA9 regulates BRCA1 expression to modulate human breast tumor phenotype. J Clin Invest 120, 1535-1550, https://doi.org/10.1172/JCI39534 (2010).

37. Pojo, M. et al. A transcriptomic signature mediated by HOXA9 promotes human glioblastoma initiation, aggressiveness and resistance to temozolomide. Oncotarget 6, 7657-7674, https://doi.org/10.18632/oncotarget.3150 (2015).

38. Ko, S. Y., Ladanyi, A., Lengyel, E. \& Naora, H. Expression of the homeobox gene HOXA9 in ovarian cancer induces peritoneal macrophages to acquire an M2 tumor-promoting phenotype. The American journal of pathology 184, 271-281, https://doi. org/10.1016/j.ajpath.2013.09.017 (2014).

39. Ko, S. Y. et al. HOXA9 promotes ovarian cancer growth by stimulating cancer-associated fibroblasts. J Clin Invest 122, 3603-3617, https://doi.org/10.1172/jci62229 (2012).

\section{Acknowledgements}

We would like to show our appreciation for professor Chul-Ho Kim (Department of Otolaryngology and Department of Molecular Science and Technology, Ajou University, Korea) for thyroid cell lines, Nthy-Ori 3-1, TPC1, and BHP10-3. This study was supported by grants from the National Research Foundation, Korea (NRF2017R1C1B1006395).

\section{Author Contributions}

I.S., Y.S.C. and Y.J.C. designed the research; Y.J. performed the research; Y.J., I.S., H.K.K., J.L., E.Y.S., Y.S.C. and Y.J.C. analysed the experimental results; Y.J. and Y.J.C. wrote the paper. All authors commented on and approved the manuscript.

\section{Additional Information}

Supplementary information accompanies this paper at https://doi.org/10.1038/s41598-019-43207-5.

Competing Interests: The authors declare no competing interests.

Publisher's note: Springer Nature remains neutral with regard to jurisdictional claims in published maps and institutional affiliations.

Open Access This article is licensed under a Creative Commons Attribution 4.0 International License, which permits use, sharing, adaptation, distribution and reproduction in any medium or format, as long as you give appropriate credit to the original author(s) and the source, provide a link to the Creative Commons license, and indicate if changes were made. The images or other third party material in this article are included in the article's Creative Commons license, unless indicated otherwise in a credit line to the material. If material is not included in the article's Creative Commons license and your intended use is not permitted by statutory regulation or exceeds the permitted use, you will need to obtain permission directly from the copyright holder. To view a copy of this license, visit http://creativecommons.org/licenses/by/4.0/.

(C) The Author(s) 2019 